\title{
The Advances and Challenges of CAR-NK Cells for Tumor Immunotherapy
}

\author{
Ziyun A. Ye $\mathrm{e}^{1, \mathrm{a}}$ \\ ${ }^{1}$ Department of Chemistry, Indiana University, IN 47405, USA
}

\begin{abstract}
Immunotherapies using chimeric antigen receptor (CAR)-T cells bring an encouraging vision to non-Hodgkin lymphoma patients who develop relapsed lymphoma or are unresponsive to standard chemotherapy, yet they also have limitations and drawbacks. Clinical trials have reported cases of neurotoxicity and cytokine release syndrome (CRS) accompanied by CAR-T cell therapies. To establish a more mature therapy, CAR incorporated into Natural Killer (NK) cells came into being. As a leukocyte involved in innate immunity, NK cell does not require MHC matching, making the production of allogeneic "off-the-shelf" CAR-NK cells possible. Moreover, the controllable life span of CAR-NK cells and little risk of graft-versus-host disease reduce side effects companion by CAR-T. This review provides an overview of CAR-NK design and production before delivery to patients. Different sources of NK cells are compared and the development of CAR molecule construction is introduced.
\end{abstract}

\section{Introduction}

Before the emerging of immunotherapies for nonHodgkin lymphomas (NHL), hematopoietic stem cell transplantation (HSCT) is applied to treat B-cell malignancies including chronic lymphoblastic leukemia (CLL) , acute lymphoblastic leukemia (ALL), and lymphoma, yet its capability is limited when treating patients with refractory or relapsed lymphoma after chemotherapy. The side effect of HSCT, such as graftversus-host disease (GVHD), causes inflammation and damage to host organs as well as the immune system. Chimeric antigen receptor (CAR)-T cell therapy, on the other hand, provides promising results in treating many NHL subtypes. "Chimeric" stands for a combination of single-chain variable region ( $\mathrm{scFv})$, hinge region, transmembrane domain and $\mathrm{T}$ cell activation domain. Comparing to first-generation CAR, later generations have additional co-stimulatory domains developed from CD28 or 4-1BB [1]. The co-stimulatory domain was demonstrated to promote the expansion and prolong the lifespan of CAR-T cells in lymphoma patients [2]. Genetical reengineering of $\mathrm{T}$ cells isolated from patients allows expression of CAR construct on cell surfaces. These CAR-T cells are expanded in vitro and infused back to patients, usually after conditioning chemotherapy is given.

CAR-T cells realize the possibility of $\mathrm{T}$ cell therapies independent of $\mathrm{T}$ cell receptors (TCR). CAR enables MHC-independent recognition to avoid omission of tumor cells that reduce their display by downregulating MHC molecules [3]. CAR-T cells targeting CD19 were approved by U.S. FDA for the treatment of diffuse largeB-cell lymphoma and refractory pre-B cell ALL. In a clinical trial of 243 patients treated with anti-CD19 CAR$\mathrm{T}$ cells, the objective response reached over $60 \%$ with only $20 \%$ not responded [4]. Since CD19 is not expressed on Hodgkin lymphoma and some B cell NHLs, CARs targeting alternative lymphoma-associated antigens, such as CD20, CD22 and CD30 become new areas of research [1].

While CAR-T cell therapy treating lymphoma obtained some impressive results, there are some limitations accompanied by. Lymphocytes depletion and T-cell activity enhancement can lead to neurotoxicity, in some cases fatal, potentially due to diffusion of cytokine or activated CAR-T cells crossing the blood-brain barrier [4]. Cytokine release syndrome (CRS) characterized by elevated serum levels of cytokines, cerebral edema, tachycardia, and fever was observed in treatment using CAR-T cells targeting CD19, CD22 and BCMA [3]. In addition, clinical trials reported the emergence of loss of CD19 on tumor cells as an escape mechanism, causing relapse in patients [5]. Another concern of CAR-T cells in therapy is their long persistence in circulation that may result in B-cell deficiency [6]. Tumor-associated antigens expressed on healthy tissues can be targeted by CAR-T cells, resulting in on-target/off-tumor side effect such as bone marrow suppression [7].

On the other hand, the capability of NK cells to distinguish "self" (normal cells) from "non-self" (infected cells, tumor cells) makes them appealing to tumor immunotherapy. Alloreactivity of NK cells depends on signals received from surface activatory and inhibitory receptors: NK cells do not kill when their inhibitory receptors match to MHC I molecules of "self", and their killing is activated when stressed cells downregulate MHC I molecules or overexpress activatory ligands. NK cells immunotherapy has demonstrated efficacy either when killer-cell immunoglobulin-like receptor (KIR) mismatches constitutively express ligands on tumor cells or when TRAIL-dependent/NKR-independent

${ }^{a}$ Corresponding author: yeziy@iu.edu 
cytotoxicity is induced [8]. NK cells can be activated by dendritic cells (DC), MHC-I-negative cells, IgG binding or cytokines such as type I IFNs [8]. Cytokine stimulation induces NK cells to secrete cytokines like IFN- $\gamma$, which suppresses tumor angiogenesis, and $\mathrm{TNF} \alpha$ that triggers inflammatory responses. Further understanding of the balance of inhibitory and activatory receptors, as well as their ligands, is essential for new approaches in NK cell immunotherapy.

As tumor cells have immune escape mechanisms against NK cells cytotoxicity, the need for depressing cytotoxicity expedites the improvement of CAR-NK cells, which express surface receptors that bind tumorassociated antigens like CAR-T cells do. Similar to the development of CAR-T cells, the addition of costimulatory domains to $\mathrm{scFv}$ and activation domain (usually $\mathrm{CD} 3 \zeta$, DAP10 or DAP12) can increase the efficacy of CAR-NK cells. As allogenic CAR-T cells are rejected by patient's immune system, the source of $\mathrm{T}$ cell needs to be autologous. However, since MHC matching is not a requirement of NK cell activation, CAR-NK cells can trigger target cells death through natural cytotoxicity receptors and the source can be allogeneic [9]. Further, NK cells are known to retain their graft-versus-tumor (GVT) effect while avoiding GVHD that is observed with $\mathrm{T}$ cells [10], as long as they are not administered to patients previously received allogeneic HSCT[11]. Comparing to CAR-T, mature donor CAR-NK cells are less problematic with on target/off tumor side effect because they have limited life span and are expected to be rejected after killing [12]. In addition, a suicide system can be incorporated into immature CAR-NK cells to ensure safety [12].

\section{Sources of NK cells used for generation of CAR-NK cells}

\subsection{NK92 cell line}

The most studied and widely applied source for CAR-NK cell generation is NK cell line, especially NK-92 cell line [9]. Unlike archetypal NK cells, NK-92 cells lack several activatory receptors such as CD16, NKp44, and NKp46 [9], they were also shown to lack most inhibitory KIRs, except for KIR2DL4 [13]. CAR-NK-92 cells have been designed and generated to target tumor antigens, such as CD19, CD20, HER2, ErbB2, and CD138 [9]. Among these tumor-associated antigens, ErbB2 is overexpressed in many epithelial tumors. In one study, CAR-NK-92 cells targeting ErbB2 showed specificity and efficiency in lysing ErbB2-expressing tumor cells, compared with wildtype NK-92 cells [14]. 86\% of tumor cells from breast, ovarian, and squamous cell carcinomas expressing high levels of ErbB2 were completely resistant to NK-92, yet they were effectively lysed by CAR-NK-92 cells even at low E/T ratio [14]. A study found that cells from ALL patients that are insensitive to NK-92 mediated lysis show augmented sensitivity to anti-CD19 CAR-NK-92 cells [15]. These CAR transfected NK-92 cells are also expected to have less side effect due to short life span [15].

\subsection{Peripheral blood (PB) and umbilical cord blood (UCB)}

NK cells isolated from peripheral blood (PB) and umbilical cord blood (UCB) can also be used to target leukemia. One clinical trial found them effective against Acute Myeloid Leukemia (AML) [16]. Comparing to NK-92 cell lines, NK cells from PB express more activatory receptors such as CD16, NKp44, and NKp46, increasing the strength of attacking signals. PB-NK cells are more heterogeneous, yet they do not require transfection of CD16 for ADCC [9]. However, the isolation from blood apheresis can be expensive and complicated, as NK cells need to be expanded and activated in the presence of feeder cells. Additionally, NK cells purified from PB have short life spans in vivo, usually only up to a few weeks [17], which limit their effectiveness in therapy.

Alternatively, UCB is another good source of large number of allogeneic NK cells. Its capability of being cryopreserved makes off-the-shelf product probable. NK cells from UCB have higher percentage composition and possess reduced cytotoxicity than that from $\mathrm{PB}[18,19]$. The unique NK cell progenitors in UCB but not in PB allow faster recovery of NK cells after transplantation [20]. Studies reported that artificial antigen presenting cells expressing IL-21 [21]or IL-15 [22]can be used for large-scale NK cell expansion. These expanded UCB-NK cells displayed purity of $\mathrm{CD}_{5} 6^{+} \mathrm{CD} 3^{-}$over $95 \%$ and increased perforin and granzyme $\mathrm{B}$ expression. A recent study indicated CB-NK cells expressing iC9/CAR.19/IL15 target and kill CD19-expressing cell lines and primary CLL cells effectively, and the accompanied inflammatory response can be counteracted with activation of iC9 [23]. More clinical trials are needed to assess the practicability of off-the-shelf UCB-NK cells product.

\subsection{Induced pluripotent stem cell-derived NK cells}

NK cells expressing CAR can also be derived from induced pluripotent stem cells (iPSCs) or human embryonic stem cells (hESCs). iPSC-NK cells overcome some of the limitations of NK-92, PB-NK cells and UCBNKs. Comparing to NK-92 cells, they express NKp44, NKp46, and KIRs, and they do not require irradiation before administration to patients; comparing to heterogeneous PB-NK cells, they can be homogeneous and express CAR more stably [9]. A study compares the cytotoxicity of NK cells isolated from PB and from iPSC in killing ovarian cancer cells [24], indicating that both NK cells population have significant effect on cancer cells killing. The expression of CAR can further boost the anti-tumor activity of effector NK cells. Using an xenograft model, another study showed that CAR-iPSCNK cells inhibit ovarian tumor growth more effectively than T cells, PB-NK, and iPSC-NK cells [25]. Also, CAR-iPSC-NK cells demonstrate similar anti-tumor 
activity as CAR-T cells but with lower toxicity. In general, CAR-iPSC-NK cells manifest promising capability to kill solid tumors, and their "off-the-shelf" products can be generated in quantity.

\section{CAR molecule construction for CAR- NK cell therapy}

Several generations of CAR molecules have been constructed for the improvement of cytotoxicity of NK cells. The most common form of first-generation CARs consists of a $\mathrm{scFv}$ derived from monoclonal antibody for antigen-recognition and a transmembrane $\mathrm{CD} 3 \zeta$ chain for activation signal. On the basis of the first-generation CAR, the second-generation CAR adds in a costimulatory domain, such as CD28, 4-1BB,or 2B4 (CD244). This costimulator is incorporated into the cytoplasmic portion of CAR to increase the signaling from the activation domain. 2B4 contains immunoreceptor tyrosine-based switch motifs (ITSMs) that are responsible for NK cells stimulatory pathway activation [26], and its combination with $\mathrm{CD} 3 \zeta$ enhance IFN- $\gamma$ and TNF- $\alpha$ production [27]. Similar results were obtained using 4-1BB-CD3 $\zeta$ CAR construct in NK cells. Furthermore, another study confirmed in vivo anti-tumor activity of GPC3-specific CAR-NK-92 with $\mathrm{CD} 28$ against $\mathrm{GPC}^{+}$hepatocellular carcinoma (HCC) [28]. When GPC3+ HCC cells are present, these CAR-NK cells are able to produce a large amount of IFN- $\gamma$, and their function is unaffected in hypoxic $(1 \%)$ environment. Two separate studies constructed similar CARs with CD28 to target tumorassociated ErbB2/HER2 antigen and observed efficient serial cell killing in Glioblastoma [29] and breast carcinoma [30].

To further enhance activation signals and cytokine production, the third-generation CAR includes multiple costimulatory domains selected among CD28, 2B4, 4$1 \mathrm{BB}$, and OX40 (CD134). OX40, belonging to the TNF receptor superfamily, is first identified on activated $\mathrm{T}$ cells to trigger downstream signaling, such as the activation of NK- $\mathrm{KB}$ to enhance cell survival [31]. OX40 incorporated in third-generation CAR-T cells shows increased cytokine production and cytotoxicity [32], but it has not been incorporated into CAR-NK cells yet.

\section{CAR-NK generation, culture, and manufacture}

Once a biomarker on target cells is selected and CAR molecules are designed and constructed, the next step is to deliver them to NK cells through viruses or electroporation. Both methods have been used in the generation of CAR-T cells and CAR-NK cells. NK-92 cell line has been selected extensively for genetic modification because of its high and variable transfection efficacy relative to primary NK cells [33]. A viral delivery system transduces NK cells for CAR molecules by retrovirus or its subclass, lentivirus. These viruses can convert their RNA back to DNA and insert it into the host genome, and the host NK cells then express the viral DNA coded for CAR and load CAR molecules on its cell surface. Comparing to retroviral transfection, lentiviral transfection infects not only dividing cells but also nondividing cells. Studies have shown that NK-92 cells modified by either CAR vectors can induce apoptosis of cells overexpressing ErbB2 antigen in breast cancer and glioblastoma $[14,33]$. NK cells expressing high levels of CAR are enriched by magnetic immunoselection or flow cytometric cell sorting [14, 34]. Comparing to viral transduction, electroporation transfection has lower efficiency due to large-scale cell death, but it lowers the risk of insertional mutagenesis and is technically simpler [35]. In electroporation, NK cells are exposed to an electric field and membranes are disrupted to allow entry of mRNA or plasmid DNA. Transfection with mRNA has an efficiency $(>75 \%)$ ten times higher than with DNA plasmid and modified anti-CD19 CAR-NK-92 cells effectively target lymphoid malignancies [36]. In addition, mRNA does not enter the nucleus and integrate into the genome like DNA, thus it eliminates the risk of genomic mutation and has immediate CAR expression. The purity of CAR-NK-92 cells can reach nearly absolute upon enrichment [35].

Since around $13 \%$ of lymphocytes in blood are constituted by CD3-/CD16-56+ NK cells [37], the isolation of NK cells from blood is straightforward in operation and able to reach a high purity. Immunomagnetic beads attached to anti-CD3 and antiCD56 antibodies are often used to deplete CD3 T cells and enrich CD56 cells [38]. To further expand effector NK cells ex vivo, the most productive method is to culture NK cells in the presence of cytokines IL-21 following IL-2 and irradiated autologous feeder cells in GMP-compliant media [39]. Cell expansion increases NK cell expression of cytotoxicity receptors and death ligands in vitro. On the other hand, the culture of NK cells after CAR incorporation is more critical as a large quantity of cell is needed for the clinic, and the process of enrichment of CAR-NK cells is similar to that of primary NK cells. After transduction, the expression of surface CAR is analyzed by fluorescence-activated cell sorter (FACS), and cells with high level of CAR are enriched by magnetic separation [15].

\section{Conclusion}

Based on maturing CAR-T cell immunotherapy, CARNK cell therapy develops and attracts growing attention because of its limited side effects and convenience of source. From the identification of CAR biomarker to CAR-NK clinical trial, each step needs to be optimized to enhance NK cells specificity and anti-tumor activity. Even though in vivo assays show encouraging results of CAR-NK cells targeting ALL, AML, CLL, Glioblastoma and some carcinomas, more investigation is needed to optimize CAR constructs for NK cell activation. The development of next-generation CAR with various combination of intracellular signaling domains and scFvs is critical for answering this question. In addition, the requirement of stable CAR expression and large scale "off-the-shelf" cell products needs a further comparison 
among different sources of NK cells and different CAR incorporation mechanisms.

\section{References}

1. J. N. Brudno, J. N. Kochenderfer, Nat Rev Clin Oncol 15, 31-46 (2018).

2. B. Savoldo, C. A. Ramos, E. Liu, M. P. Mims, M. J. Keating, G. Carrum, R. T. Kamble, C. M. Bollard, A. P. Gee, Z. Mei, The Journal of clinical investigation 121, 1822-6 (2011).

3. C. H. June, R. S. O'Connor, O. U. Kawalekar, S. Ghassemi, M. C. Milone, Science 359, 1361-5 (2018).

4. J. Hartmann, M. Schüßler-Lenz, A. Bondanza, C. J. Buchholz, EMBO molecular medicine 9, 1183-97 (2017).

5. S. A. Grupp, M. Kalos, D. Barrett, R. Aplenc, D. L. Porter, S. R. Rheingold, D. T. Teachey, A. Chew, B. Hauck, J. F. Wright, M. C. Milone, B. L. Levine, C. H. June, New England Journal of Medicine 368, 1509-18 (2013).

6. H. Klingemann, Oncoimmunology 3, e28147 (2014).

7. S. K. Tasian, M. Ruella, O. Shestova, Y. Li, D. L. Porter, M. Carroll, G. Danet-Desnoyers, J. Scholler, S. A. Grupp, C. H. June, M. Kalos, Blood 122, 143(2013)

8. L. Zamai, C. Ponti, P. Mirandola, G. Gobbi, S. Papa, L. Galeotti, L. Cocco, M. Vitale, The Journal of Immunology 178, 4011-6 (2007).

9. D. L. Hermanson, D. S. Kaufman, Front Immunol 6, 195 (2015).

10. J. A. Olson, D. B. Leveson-Gower, S. Gill, J. Baker, A. Beilhack, R. S. Negrin, Blood 115, 4293-301 (2010).

11. N. N. Shah, K. Baird, C. P. Delbrook, T. A. Fleisher, M. E. Kohler, S. Rampertaap, K. Lemberg, C. K. Hurley, D. E. Kleiner, M. S. Merchant, S. Pittaluga, M. Sabatino, D. F. Stroncek, A. S. Wayne, H. Zhang, T. J. Fry, C. L. Mackall, Blood 125, 784-92 (2015).

12. W. Glienke, R. Esser, C. Priesner, J. D. Suerth, A. Schambach, W. S. Wels, M. Grez, S. Kloess, L. Arseniev, U. Koehl, Front Pharmacol 6, 21 (2015).

13. D. N. Burshtyn, A. M. Scharenberg, N. Wagtmann, S. Rajagopalan, K. Berrada, T. Yi, J. P. Kinet, E. O. Long, Immunity 4, 77-85 (1996).

14. C. Uherek, T. Tonn, B. Uherek, S. Becker, B. Schnierle, H.-G. Klingemann, W. Wels, Blood 100, 1265-73 (2002).

15. A. Romanski, C. Uherek, G. Bug, E. Seifried, H. Klingemann, W. S. Wels, O. G. Ottmann, T. Tonn, Journal of cellular and molecular medicine 20, 1287 94 (2016).

16. Y. Yang, O. Lim, T. M. Kim, Y.-O. Ahn, H. Choi, H. Chung, B. Min, J. H. Her, S. Y. Cho, B. Keam, S.-H. Lee, D.-W. Kim, Y. K. Hwang, D. S. Heo, Cancer Immunology Research 4, 215-24 (2016).
17. Y. Hu, Z.-g. Tian, C. Zhang, Acta Pharmacologica Sinica 39, 167 (2017).

18. P. K. Kotylo, J. C. Baenzinger, M. C. Yoder, W. A. Engle, C. D. Bolinger, American Journal of Clinical Pathology 93, 263-6 (1990).

19. A. Sarvaria, D. Jawdat, J. A. Madrigal, A. Saudemont, Frontiers in immunology 8, 329 (2017).

20. S. Cooley, V. McCullar, R. Wangen, T. L. Bergemann, S. Spellman, D. J. Weisdorf, J. S. Miller, Blood 106, 4370-6 (2005).

21. N. Shah, B. Martin-Antonio, H. Yang, S. Ku, D. A. Lee, L. J. N. Cooper, W. K. Decker, S. Li, S. N. Robinson, T. Sekine, S. Parmar, J. Gribben, M. Wang, K. Rezvani, E. Yvon, A. Najjar, J. Burks, I. Kaur, R. E. Champlin, C. M. Bollard, E. J. Shpall, PLOS ONE 8, e76781 (2013).

22. J. Ayello, J. Hochberg, A. Flower, Y. Chu, L. V. Baxi, W. Quish, C. van de Ven, M. S. Cairo, Experimental hematology 46, 38-47 (2017).

23. E. Liu, Y. Tong, G. Dotti, H. Shaim, B. Savoldo, M. Mukherjee, J. Orange, X. Wan, X. Lu, A. Reynolds, Leukemia 32, 520 (2018).

24. D. L. Hermanson, L. Bendzick, L. Pribyl, V. McCullar, R. I. Vogel, J. S. Miller, M. A. Geller, D. S. Kaufman, Stem Cells 34, 93-101 (2016).

25. Y. Li, D. L. Hermanson, B. S. Moriarity, D. S. Kaufman, Cell stem cell 23, 181-92. e5 (2018).

26. B. A. Garni-Wagner, A. Purohit, P. A. Mathew, M. Bennett, V. Kumar, The Journal of Immunology 151, 60-70 (1993).

27. O. Oberschmidt, S. Kloess, U. Koehl, Frontiers in immunology 8, 654- (2017).

28. M. Yu, H. Luo, M. Fan, X. Wu, B. Shi, S. Di, Y. Liu, Z. Pan, H. Jiang, Z. Li, Molecular Therapy 26, 36678 (2018).

29. C. Zhang, M. C. Burger, L. Jennewein, S. Genßler, K. Schönfeld, P. Zeiner, E. Hattingen, P. N. Harter, M. Mittelbronn, T. Tonn, Journal of the National Cancer Institute 108, djv375 (2015).

30. K. Schönfeld, C. Sahm, C. Zhang, S. Naundorf, C. Brendel, M. Odendahl, P. Nowakowska, H. Bönig, U. Köhl, S. Kloess, Molecular therapy 23, 330-8 (2015).

31. M. Croft, T. So, W. Duan, P. Soroosh, Immunological reviews 229, 173-91 (2009).

32. M. A. Pulè, K. C. Straathof, G. Dotti, H. E. Heslop, C. M. Rooney, M. K. Brenner, Molecular Therapy 12, 933-41 (2005).

33. G. Suck, M. Odendahl, P. Nowakowska, C. Seidl, W. S. Wels, H. G. Klingemann, T. Tonn, Cancer immunology, immunotherapy 65, 485-92 (2016).

34. S. Oelsner, M. E. Friede, C. Zhang, J. Wagner, S. Badura, P. Bader, E. Ullrich, O. G. Ottmann, H. Klingemann, T. Tonn, Cytotherapy 19, 235-49 (2017).

35. H. Klingemann, Oncoimmunology 3, e28147 (2014). 
36. L. Boissel, M. Betancur, W. S. Wels, H. Tuncer, H. Klingemann, Leukemia research 33, 1255-9 (2009).

37. W. M. Comans-Bitter, R. de Groot, R. van den Beemd, H. J. Neijens, W. C. Hop, K. Groeneveld, H. Hooijkaas, J. J. van Dongen, The Journal of pediatrics 130, 388-93 (1997).

38. U. Koehl, C. Brehm, S. Huenecke, S.-Y. Zimmermann, S. Kloess, M. Bremm, E. Ullrich, J. Soerensen, A. Quaiser, S. Erben, C. Wunram, T. Gardlowski, E. Auth, T. Tonn, C. Seidl, S. MeyerMonard, M. Stern, J. Passweg, T. Klingebiel, P. Bader, D. Schwabe, R. Esser, Frontiers in oncology 3, 118- (2013).

39. S. Klöß, O. Oberschmidt, M. Morgan, J. Dahlke, L. Arseniev, V. Huppert, M. Granzin, T. Gardlowski, N. Matthies, S. Soltenborn, Human gene therapy 28, 897-913 (2017). 